\title{
CORRECTION
}

\section{Author Correction: Threshold for neural tube defect risk by accumulated singleton loss-of-function variants}

Zhongzhong Chen ${ }^{1,2}$, Yunping Lei ${ }^{3}$, Yufang Zheng iD $^{1,2,4}$, Vanessa Aguiar-Pulido ${ }^{5}$, M. Elizabeth Ross ${ }^{5}$, Rui Peng ${ }^{1}$, Li Jin ${ }^{1,2}$, Ting Zhang ${ }^{6}$, Richard H. Finnell ${ }^{3}$ and Hongyan Wang ${ }^{1,2,7,8}$

Cell Research (2019) 29:776; https://doi.org/10.1038/s41422-019-0201-4

Correction to: Cell Research https://doi.org/10.1038/s41422-0180061-3, published online 05 July 2018

We apologize for one error that we just found in the paper published in 2018, October issue. There were mistakes in the institutional affiliation of co-authors Yunping Lei and Richard $\mathrm{H}$. Finnell. Both authors were only affiliated to \#3, Departments of Molecular and Cellular Biology and Medicine, Baylor College of Medicine, Houston, TX 77030, USA. There is no co-authorship with the affiliation \#4. This correction does not affect the description of the results or the conclusion of this work. We apologize for not detecting them before publication and any inconvenience caused. The correct affiliations of authors should be as follows:

Zhongzhong Chen ${ }^{1,2}$, Yunping Lei ${ }^{3}$, Yufang Zheng ${ }^{1,2,4}$, Vanessa Aguiar-Pulido ${ }^{5}$, M. Elizabeth Ross ${ }^{5}$, Rui Peng ${ }^{1}$, Li Jin ${ }^{1,2}$, Ting Zhang ${ }^{6}$, Richard H. Finnell ${ }^{3}$ and Hongyan Wang ${ }^{1,2,7,8}$

\begin{abstract}
${ }^{1}$ Obstetrics and Gynecology Hospital, State Key Laboratory of Genetic Engineering at School of Life Sciences, Institute of Reproduction and Development, Fudan University, Shanghai 200011, China; ${ }^{2}$ Key Laboratory of Reproduction Regulation of NPFPC, Collaborative Innovation Center of Genetics and Development, Fudan University, Shanghai 200032, China; ${ }^{3}$ Departments of Molecular and Cellular Biology and Medicine, Baylor College of Medicine, Houston, TX 77030, USA; ${ }^{4}$ Institute of Developmental Biology \& Molecular Medicine, Fudan University, Shanghai 200433, China; ${ }^{5}$ Center for Neurogenetics, Weill Cornell Medicine, New York, NY 10021, USA; ${ }^{6}$ Capital Institute of Pediatrics, Beijing 100020, China; ${ }^{7}$ Children's Hospital, Fudan University, 399 Wanyuan Road, Shanghai 201102, China; ${ }^{8}$ Institutes of Biomedical Sciences, Fudan University, Shanghai 200032, China
\end{abstract}

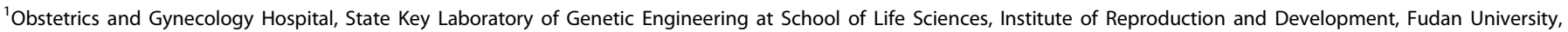

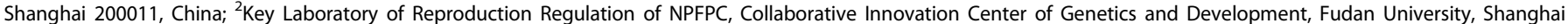

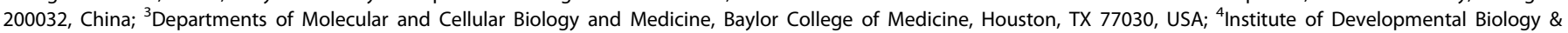

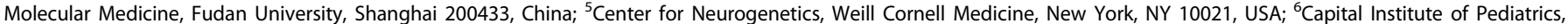

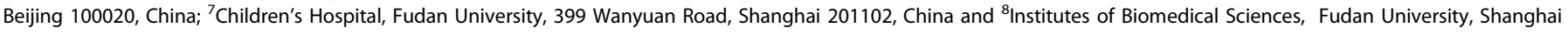
200032, China

Correspondence: Ting Zhang (zhangtingcv@126.com) or Richard H. Finnell (Richard.Finnell@bcm.edu) or Hongyan Wang (wanghy@fudan.edu.cn)

These authors contributed equally: Zhongzhong Chen, Yunping Lei and Yufang Zheng
}

Published online: 25 July 2019 